\title{
Transient hot UV spectra in the collisional deactivation of highly excited trans-stilbene in liquid solvents
}

\author{
L. Nikowa, D. Schwarzer, J. Troe \\ Max-Planck-Institut für biophysikalische Chemie Am Faßberg, D-37077 Göttingen, Germany
}

Received 26 October 1994; in final form 22 November 1994

\begin{abstract}
Collisional energy transfer of vibrationally highly excited ground state trans-stilbene, formed by isomerization and internal conversion of electronically excited cis-stilbene, was investigated in liquid $n$-pentane and methanol. The temporal evolution of the trans-stilbene absorption spectrum between 295 and $310 \mathrm{~nm}$ was reconstructed from wavelength dependent fluorescence yields. Energy-loss profiles were generated using separate shock wave studies of stilbene high temperature spectra for calibration. Most of the vibrational energy of excited trans-stilbene is transferred to the solvent within $1-2 \mathrm{ps}$. Subsequently, the cooling process continues on a $10 \mathrm{ps}$ timescale due to local heating of the liquid environment.
\end{abstract}

\section{Introduction}

In contrast to the gas phase [1], the mechanism of collisional energy transfer of vibrationally highly excited polyatomic molecules in liquid solvents is far from being understood. Although several studies on this subject exist ([2-8] and review [9] with references cited therein), basic questions remain unanswered:

(i) Does vibrational energy transfer in the liquid phase follow the same mechanism as in the gas phase or do fundamental differences arise?

(ii) Can binary collisions be identified and does the averaged energy $\langle\Delta E\rangle$ transferred per binary collision change from gas phase collision to dense liquid phase encounter conditions?

(iii) Does the collision number $Z$ increase from gas to compressed liquid phase proportional to the inverse $D^{-1}$ of the solvent self-diffusion coefficient?
We previously have used $D^{-1}$ scales to represent isomerization or atom recombination rate constants over the gas-liquid transition [10,11]. Using $D^{-1}$ scales for vibrational relaxation in liquids, i.e. for collisional energy transfer of only slightly excited molecules, has also been suggested on theoretical grounds [12].

Vibrationally highly excited molecules are often generated by light absorption into excited electronic states followed by rapid internal conversion to the electronic ground state [1]. The loss of vibrational energy then can directly be monitored following UV/VIS absorption signals which are converted into energy/temperature-time profiles, provided that the energy/temperature dependence of the absorption coefficient is known [13]. In the present study we will show that highly excited trans-stilbene is a suitable model system to study collisional deactivation by this technique. Following basically the same 
method as in Refs. [7,14], excited molecules were prepared by irradiating cis-stilbene in solution with laser pulses at $310 \mathrm{~nm}$. The electronically excited cis-stilbene rapidly isomerizes and internally converts into a mixture of vibrationally highly excited electronic ground state cis- and trans-stilbene as well as dihydrophenanthrene molecules. Out of this mixture, the trans-stilbene molecules can be detected selectively by exciting their fluorescence. By using hot thermal spectra studied in shock tube experiments, the fluorescence signals can be converted into energy/temperature-time profiles. First experimental results were presented in Ref. [15].

\section{Experimental technique}

The optical pulses required for the present study were generated by a laser system based on a colliding pulse mode-locked dye laser. Its output pulses were amplified in a three stage Nd:YAG pumped dye amplifier [16] at a $30 \mathrm{~Hz}$ repetition rate. After frequency doubling in a potassium dihydrogen phosphate (KDP) crystal, pump pulses at a wavelength of $310 \mathrm{~nm}$ and $\approx 100 \mathrm{fs}$ width were obtained. In some experiments, $70 \%$ of the pulse energy was used to pump cis-stilbene to its first electronic singlet state whereas $30 \%$ of the energy were used to probe the trans-stilbene fluorescence. In order to generate probe pulses at 295,325 , and $330 \mathrm{~nm}$, the remaining laser beam at $620 \mathrm{~nm}$ was focused into a water cell generating a white light continuum. A spectral band at twice the desired wavelength was amplified and frequency doubled in a KDP crystal. Pump and probe pulses were recombined and collinearly focused into the sample cell. The respective energies were approximately 1 and $0.5 \mu \mathrm{J}$. In order to eliminate effects of overall rotational relaxation (see Ref. [59] in Ref. [8]), the plane of polarisation of the probe pulse by means of a zeroth-order half-wave plate, was adjusted to $54.7^{\circ}$ with respect to the pump. The trans-stilbene fluorescence then was detected by a photomultiplier tube parallel to the pump pulse polarisation through an interference filter centered at $352 \mathrm{~nm}$ (bandwidth: $22 \mathrm{~nm}$ ). Measurements were performed in a $2 \mathrm{~mm}$ quartz flow cell. Cisstilbene was purchased from Heraeus and purified by

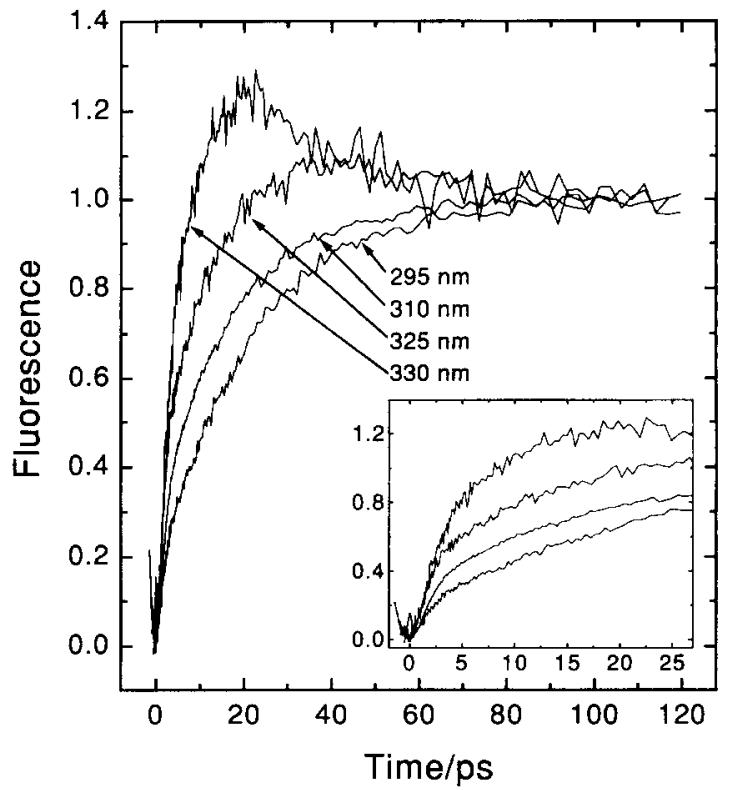

Fig. 1. Wavelength dependence of probe fluorescence signals from vibrationally highly excited trans-stilbene in liquid $n$-pentane (the insert enlargens the first stage of the process; fluorescence signals are normalized to the final level).

column chromatography. Solvents (J.T. Baker) were used without purification.

\section{Results and discussion}

Fig. 1 shows a series of normalized fluorescence-time profiles for highly excited trans-stilbene in liquid $n$-pentane at 1 bar and $298 \mathrm{~K}$ obtained with different probe wavelengths. As observed earlier for liquid $\mathrm{CO}_{2}$ [15], there is an initial rapid rise of the fluorescence signal of about 3 ps duration which has been expanded in the insert of Fig. 1. The relative amplitude of this component increases with increasing probe wavelength. Subsequently, the signals rise more slowly. Whereas at probe wavelengths of 295 and $310 \mathrm{~nm}$ a monotonous increase of the fluorescence to a final value is observed, at probe wavelengths of 325 and $330 \mathrm{~nm}$ the signals reach maxima at $\approx 40$ and $\approx 20$ ps respectively before they decay to a final value. Similar signals were obtained in liquid methanol (see Fig. 2). Here the fast initial increase of the trans-stilbene fluorescence signal ends 


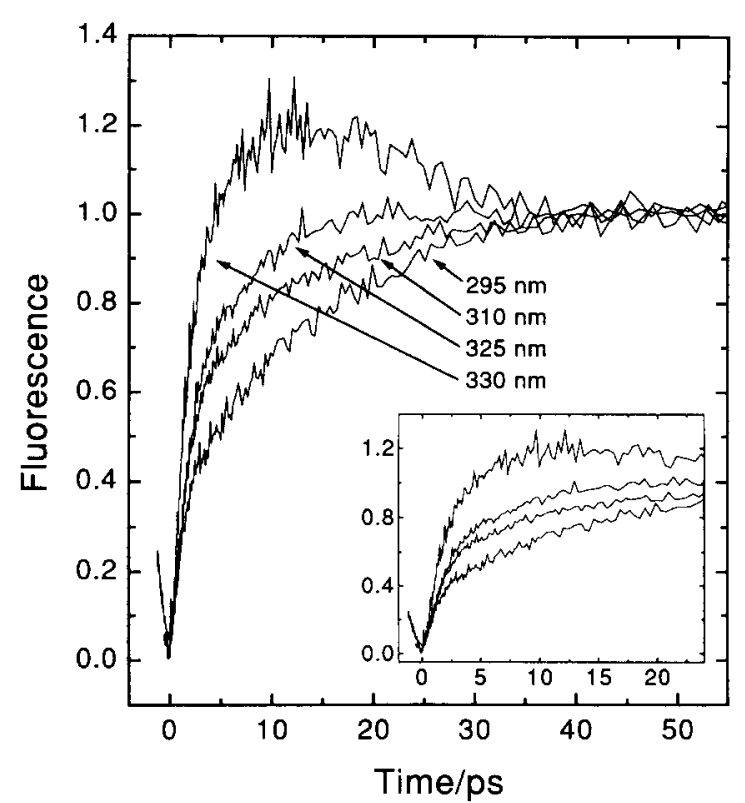

Fig. 2. As Fig. 1, in liquid methanol.

at approximately $2.5 \mathrm{ps}$, before the slower increase sets in. The amplitude of this fast component is by a factor of $\approx 1.5$ larger than in $n$-pentane for a given probe wavelength. For the probe wavelengths 295 , 310 , and $325 \mathrm{~nm}$ the signals increase monotonously; for $330 \mathrm{~nm}$ a maximum at $12 \mathrm{ps}$ is observed.

In the following we describe the procedure of converting the recorded fluorescence signals $S(t)$ into energy-loss profiles. We assume that only trans-stilbene molecules in the electronic ground state can be excited to the fluorescing state which is monitored by our detection system. Denoting the distribution function of the vibrational energy $E$ of trans-stilbene in the electronic ground state by $f(E$, $t)$ and the probe photon by $h \nu$, the fluorescence intensity is assumed to be proportional to the convolution

$S(t) \propto \int f(E, t) \epsilon(E) \Phi(E+h \nu) \mathrm{d} E$.

The absorption coefficient $\epsilon(E)$ is related to a temperature-dependent absorption coefficient $\epsilon(T)$. We have shown earlier $[13,17]$ that the 'canonically hot' and 'microcanonically hot' UV absorption spectra of a polyatomic molecule are nearly the same, if the average energies $\langle E\rangle$ of the ensembles are iden- tical. For this reason, we have measured absorption coefficients of trans-stilbene in shock waves up to temperatures of $1500 \mathrm{~K}$ [18]. Apart from a solvent shift, $\epsilon(E)$ can be well characterized up to vibrational energies $E$ of $40000 \mathrm{~cm}^{-1}$ in this way.

The fluorescence quantum yield $\Phi$ from the $S_{1}$ state at a total (electronic plus vibrational) energy $E+h \nu$ is governed by the competition between collisional stabilization and unimolecular isomerization in the $S_{1}$ state. The quantum yield for such a photoactivation system can be modelled [19], if the specific rate constants $k(E)$ for the unimolecular reaction are known and the collisional energy transfer can be characterized. Under our experimental conditions the collisional stabilization process is much faster than the isomerization, so that $\Phi$ becomes energy independent. This assumption is supported by the observation that the stationary quantum yield for trans-stilbene fluorescence was found to be independent of excitation wavelength up to $S_{1}$ excess energies of at least $10000 \mathrm{~cm}^{-1}$ [20]. We will come back to this point later on. The considerations outlined so far simplify relation (1) in such a way that the fluorescence yield depends only on the absorption coefficient, i.e. $S(t)$ directly probes the absorption coefficient $\epsilon(T)$ of trans-stilbene during the energy loss process.

For a complete analysis of the signals, the following additional aspects have to be taken into account. After the pump-pulse has reached the sample there is a short initial phase during which cis-stilbene in the $S_{1}$ state approaches a perpendicular conformation where internal conversion takes place and highly excited trans-stilbene in the $S_{0}$ state is formed. In addition, the vibrational energy has to be spread over all vibrational modes by intramolecular vibrational redistribution, before a quasiequilibrium absorption coefficient is established which is related to $\epsilon(T)$. Since the isomerization alone takes 1 ps in $n$-pentane and 0.5 ps in methanol (see Ref. [21] and references cited therein) and the following processes occur on a similar timescale, we assign the first stage of the observed signals to the formation of hot $\mathrm{S}_{0}^{*}$ transstilbene molecules. Their initial energy can be identified with the sum of the photon energy of the pump-pulse, the room temperature thermal energy of cis-stilbene and the energy difference between the cis- and trans-isomers at $0 \mathrm{~K}$. Using vibrational 
frequencies calculated by Warshel [22], this energy can be converted into a vibrational temperature of $\approx 1300 \mathrm{~K}$.

In the next step of our analysis we checked whether the observed wavelength dependence of the fluorescence signals is consistent with thermal hot trans-stilbene spectra obtained from shock wave experiments. Fig. 3 shows absorption spectra of transstilbene in liquid $n$-pentane at room temperature (solid line) and in the gas phase at $1300 \mathrm{~K}$ (dashed line, from Ref. [18]). For the $1300 \mathrm{~K}$ spectrum, a solvent red shift of $15 \mathrm{~nm}$ for the transition from gaseous to liquid $n$-pentane was taken into account in Fig. 3. If the fluorescence intensity is proportional to the absorption coefficient $\epsilon(T)$, the time dependence of the signals should depend on the probe wavelength in the following way: at wavelengths shorter than $\approx 325 \mathrm{~nm}$, where $\epsilon(1300 \mathrm{~K})<\epsilon(298$ $\mathrm{K}$ ), a fast increase of the fluorescence yield due to the preparation of hot trans-stilbene followed by a slower increase to a final value due to the stepwise energy loss is expected. The amplitude ratio of both

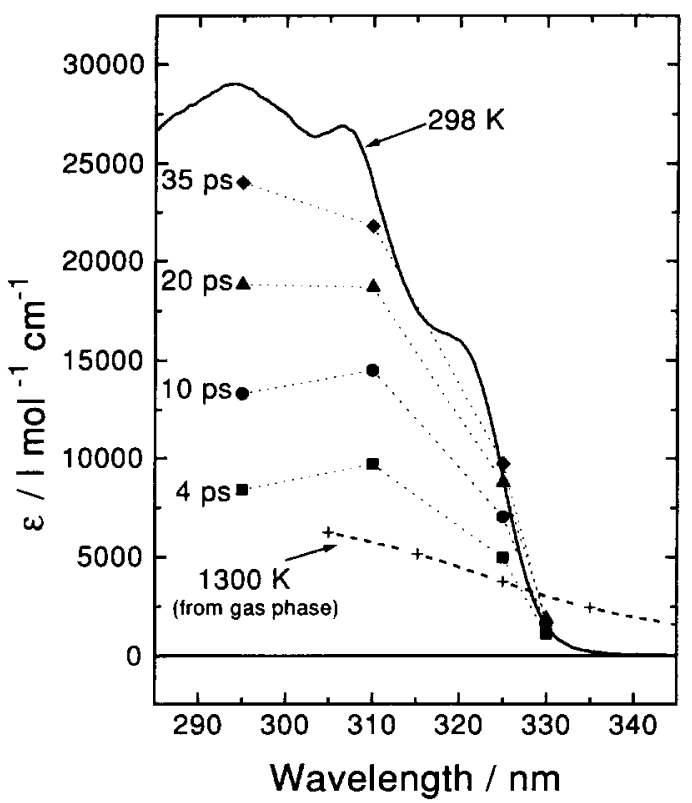

Fig. 3. Absorption spectra of trans-stilbene (full line: in liquid $n$-pentane at $298 \mathrm{~K}$; dashed line: gas phase at $1300 \mathrm{~K}$, displaced by a solvent shift of $15 \mathrm{~nm}$ to the red; dotted lines: reconstructed from fluorescence signals in $n$-pentane at the indicated time delays).

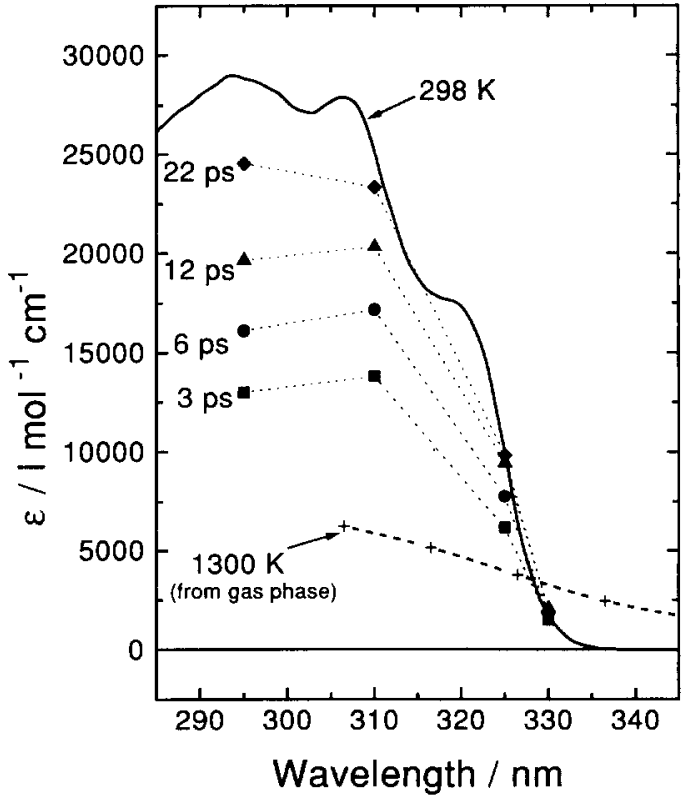

Fig. 4. Absorption spectra of trans-stilbene (full line: in liquid methanol at $298 \mathrm{~K}$; dashed line: gas phase at $1300 \mathrm{~K}$, displaced by a solvent shift of $16.5 \mathrm{~nm}$ to the red; dotted lines: reconstructed from fluorescence signals in methanol at the indicated time delays).

contributions to the signal should depend on the ratio $\epsilon(1300 \mathrm{~K}) / \epsilon(298 \mathrm{~K})$ and increase with increasing probe wavelength. Probing the fluorescence at the very red edge of the absorption spectrum more complicated signals should be observed: between the initial and the final signals a maximum is expected, when $\epsilon(1300 \mathrm{~K})>\boldsymbol{\epsilon}(298 \mathrm{~K})$. These predictions where found to be in complete agreement with our experimental results (see Figs. 1 and 2) which confirms our assumption that the fluorescence yield indeed provides a direct measure of $\epsilon(T)$. The transition from a purely monotonous increase of the signal to the appearance of a maximum is very sensitive to the probe wavelength. Since the red shift of the trans-stilbene absorption spectrum in methanol is slightly larger than in $n$-pentane $(16.5 \mathrm{~nm}$ with respect to the gas phase, see Fig. 4), at the probe wavelength $325 \mathrm{~nm}$ in contrast to $n$-pentane no maximum is observed. Maxima in the fluorescence signals would not be observed, if $\epsilon(T)$ were constant and the fluorescence yield governed solely by $\Phi(E)$, i.e. the competition between collisional stabilization 
and unimolecular isomerization in the $S_{1}$ state of trans-stilbene, as was the basic assumption in the analysis of Refs. [7,8]. It should be mentioned that our observations of profiles, which at some wavelengths show maxima whereas this is not the case at other wavelengths, are completely analogous to observations in the study of collisional energy loss of gaseous $\mathrm{CS}_{2}$ [23].

From the normalized fluorescence signals one can calculate the absorption coefficients at various times using $\epsilon(t, \lambda)=\epsilon(298 \mathrm{~K}, \lambda) S(t, \lambda)$, and reconstruct the time dependence of the trans-stilbene spectrum in its cooling process as presented for $n$-pentane and methanol in Figs. 3 and 4, respectively. The logarithm of the absorption coefficient of trans-stilbene at wavelengths around $295 \mathrm{~nm}$ in the gas phase was found to show a linear dependence on the inverse of the temperature (see Ref. [18]). Accounting for the solvent shift of the absorption spectrum in liquid $n$-pentane this temperature dependence can be represented by $\log \left[\epsilon(T) / \ell \mathrm{mol}^{-1} \mathrm{~cm}^{-1}\right]=243.7 \mathrm{~K} / T$ +3.557 at $310 \mathrm{~nm}$. Based on this calibration of the absorption coefficient, the fluorescence signal for trans-stilbene in $n$-pentane at a probe wavelength of $310 \mathrm{~nm}$ was converted into a temperature-time profile such as shown in Fig. 5 (upper trace). Since the first $3 \mathrm{ps}$ are obscured by the processes leading to hot trans-stilbene these data points are not shown in the plot. Results for vibrationally highly excited trans-stilbene in methanol probed at $310 \mathrm{~nm}$ are also shown in Fig. 5 (lower trace). Here the relation $\log \left[\epsilon(T) / \ell \mathrm{mol}^{-1} \mathrm{~cm}^{-1}\right]=260.1 \mathrm{~K} / T+3.526$ was used to convert quantum yields into temperatures. The energy-loss profiles or the corresponding cooling curves could be represented by biexponential decay functions of the form $T=298 \mathrm{~K}+$ $A \exp \left(-t / \tau_{1}\right)+(1300 \mathrm{~K}-298 \mathrm{~K}-A) \exp (-t /$ $\left.\tau_{2}\right)$. Fit parameters of $A=770 \mathrm{~K}, \tau_{1}=2.1 \mathrm{ps}, \tau_{2}=$ $12.9 \mathrm{ps}$, and $A=876 \mathrm{~K}, \tau_{1}=0.96 \mathrm{ps}, \tau_{2}=8.7 \mathrm{ps}$ were obtained for $n$-pentane and methanol, respectively. Both curves show that most of the internal energy is lost within the first picoseconds, i.e. parallel to the formation of hot trans-stilbene. After that, a slower decrease of the temperature sets in. In a more detailed analysis, the formation and cooling processes have to be separated by deconvolution to get the actual cooling curves. The corresponding analysis will be presented in a subsequent article [24]. The

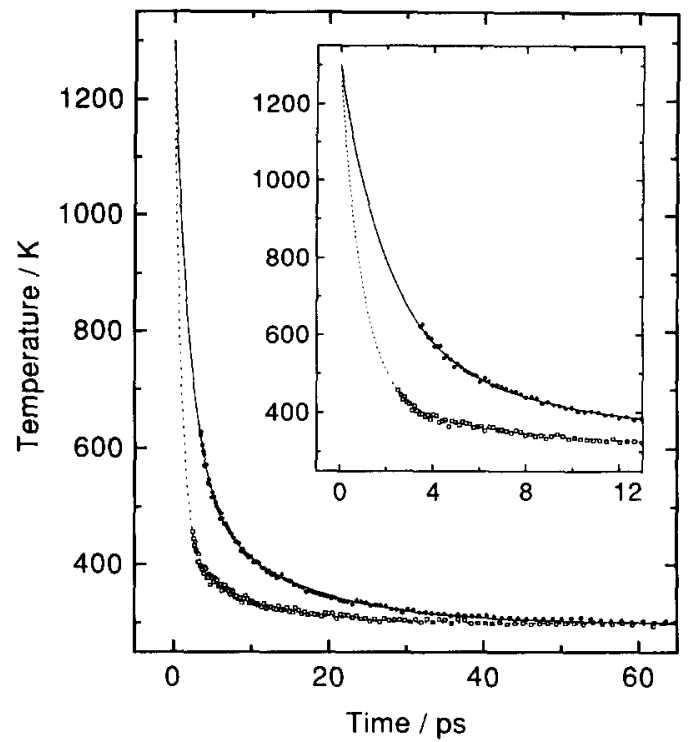

Fig. 5. Deactivation curves for trans-stilbene in liquid $n$-pentane (O) and liquid methanol ( $\square$ ) at 1 bar and $298 \mathrm{~K}$ (the insert enlargens the first stage of the process; biexponential fits of the form $T=298 \mathrm{~K}+A^{*} \exp \left(-t / \tau_{1}\right)+(1300 \mathrm{~K}-298 \mathrm{~K}-A)^{*}$ $\times \exp \left(-t / \tau_{2}\right)$ with $A=770 \mathrm{~K}, \tau_{1}=2.1 \mathrm{ps}, \tau_{2}=12.9 \mathrm{ps}$ for $n$-pentane (- ) and $A=876 \mathrm{~K}, \tau_{1}=0.96 \mathrm{ps}, \tau_{2}=8.7 \mathrm{ps}$ for methanol (...) are shown).

fast initial decay of the internal energy within 2 ps can be reproduced using $\langle\Delta E\rangle$ values known from the gas phase, and binary collision numbers $\mathrm{Z}$ scaled up to liquid densities on the basis $Z \propto D^{-1}$. The slower decrease of the internal temperature at later times probably is caused by the local heating of the liquid environment which decreases the effective $\langle\Delta E\rangle$ values. A binary collision energy transfer model accounting for the local heating of the liquid environment will be presented in Ref. [24] as well.

\section{Conclusion}

We have shown that trans-stilbene, formed by isomerization and internal conversion of electronically excited cis-stilbene, is a promising model system for studying the mechanism of collisional energy transfer of vibrationally highly excited polyatomic molecules in the liquid phase. The shape of transient fluorescence signals obtained in $n$-pentane and 
methanol for various probe wavelengths in the range $295-330 \mathrm{~nm}$ is consistent with hot thermal spectra from shock wave experiments provided that the fast increase of the fluorescence yield in the first few picoseconds is attributed to the formation process leading to hot trans-stilbene. A reconstruction of the time dependence of the hot vibrational spectra of trans-stilbene as well as a conversion of the fluorescence signals into energy-loss profiles is possible. The analysis demonstrates that most of the internal energy is lost parallel to the formation process of trans-stilbene, i.e. within 1-2 ps. Subsequently, the cooling slows down due to local heating of the liquid environment. A detailed analysis of the pressure dependence of vibrational energy transfer of transstilbene in liquid solvents together with a model describing the cooling process will be given in Ref. [22].

\section{Acknowledgements}

We thank Dr. W. Kühnle for purification of the cis-stilbene samples and Dr. J. Schroeder for helpful discussions.

\section{References}

[1] H. Hippler and J. Troe, in: Bimolecular collisions, eds. M.N.R. Ashfold and J.E. Baggott, Advances in gas-phase photochemistry and kinetics (Royal Society of Chemistry, London, 1989) p. 209.
[2] F. Wondrazek, A. Seilmeier and W. Kaiser, Chem. Phys. Letters 104 (1984) 121.

[3] A. Seilmeier, P.O.J. Scherer and W. Kaiser, Chem. Phys. Letters 105 (1984) 140.

[4] U. Sukowski, A. Seilmeier, T. Elsaesser and S.F. Fischer, J. Chem. Phys. 93 (1990) 4094.

[5] H. Miyasaka, M. Hagihara, T. Okada and N. Mataga, Chem. Phys. Letters 188 (1992) 259.

[6] K.E. Schultz, D.J. Russel and C.B. Harris, J. Chem. Phys. 97 (1992) 5431.

[7] R.J. Sension, S.T. Repinec and R.M. Hochstrasser, J. Chem. Phys. 93 (1990) 9185;

[8] R.J. Sension, S.T. Repinec, A.Z. Szarka and R.M. Hochstrasser, J. Chem. Phys. 98 (1993) 6291.

[9] T. Elsaesser and W. Kaiser, Ann. Rev. Phys. Chem. 42 (1991) 83.

[10] B. Otto, J. Schroeder and J. Troe, J. Chem. Phys. 81 (1984) 202.

[11] G. Maneke, J. Schroeder, J. Troe and F. VoB, Ber. Bunsenges. Physik. Chem. 89 (1985) 896.

[12] R. Zwanzig, J. Chem. Phys. 34 (1969) 1931.

[13] L. Brouwer, H. Hippler, L. Lindemann and J. Troe, J. Phys. Chem. 89 (1985) 4608.

[14] H. Petek, Y. Fujiwara, D. Kim and K. Yoshihara, J. Am. Chem. Soc. 110 (1988) 6269.

[15] L. Nikowa, J. Schroeder, D. Schwarzer and J. Troe, Ber. Bunsenges. Physik. Chem. 98 (1994) 262.

[16] Th. Bultmann, D. Bingemann, N. Ernsting, D. Schwarzer and L. Nikowa, to be published.

[17] M. Heymann, H. Hippler, H.J. Plach and J. Troe, J. Chem. Phys. 87 (1987) 3867.

[18] S. Frisch, H. Hippler and J. Troe, Z. Physik. Chem., in press (1994).

[19] J. Troe, J. Phys. Chem. 87 (1983) 1800.

[20] R.M. Hochstrasser, Can. J. Chem. 37 (1959) 1367.

[21] L. Nikowa, D. Schwarzer, J. Troe and J. Schroeder, J. Chem. Phys. 97 (1992) 4827.

[22] A. Warshel, J. Chem. Phys. 62 (1975) 214.

[23] J.E. Dove, H. Hippler and J. Troe, J. Chem. Phys. 82 (1985) 1907.

[24] L. Nikowa, D. Schwarzer and J. Troe, to be published. 